The proportion of males increased as severity increased. The percentage of males who had no, mild, moderate or severe OSA was $64.6 \%, 72.0 \%, 86.0$ and $87.0 \%$, respectively.

Mean age was significantly lower $(\mathrm{P}<0.001)$ in the no OSA compared to all 3 OSA categories. Those with moderate OSA were also significantly older $(\mathrm{P}<0.05)$ than those with mild OSA.

Those with severe OSA had a significantly higher average BMI compared to all other categories $(\mathrm{P}<0.05)$.

Average neck circumference significantly increased with severity of OSA.

There was no significant difference in average ESS between groups.

Conclusion Seventy-five percent of pre-operative patients with a STOP-BANG score $\geq 3$ had some degree of OSA. The severity of OSA was dependent on gender, age, BMI and neck circumference. ESS was not helpful in predicting presence or severity of OSA.

\section{P021 NON-INVASIVE VENTILATION FOR OBESE PATIENTS WITH OSA FAILING REGULAR CPAP: A PROSPECTIVE OBSERVATIONAL COHORT STUDY}

\footnotetext{
${ }^{1,2}$ Athanasius Ishak*, ${ }^{2}$ Michelle Ramsay, ${ }^{1,2}$ Nicholas Hart, ${ }^{1,2}$ Joerg Steier. ${ }^{1}$ King's College London Faculty of Life Sciences and Medicine, Centre for Human and Applied Physiological Science (CHAPS), London, UK; ' 'Guy's and St Thomas' NHS Foundation Trust Lane Fox Respiratory Unit, London, UK
}

\subsection{6/bmiresp-2019-bssconf.21}

Introduction Continuous positive airway pressure (CPAP) therapy is the standard treatment for obstructive sleep apnoea (OSA), however, patients frequently have sub-optimal longterm adherence. We hypothesised that bilevel positive airway pressure (BPAP) therapy may improve adherence and outcomes in patients who cannot tolerate CPAP.

Methods Patients with OSA who had sub-optimal CPAP usage ( $<4$ hours/day) and were referred to our sleep centre between 2014-2017 for BPAP were included. Age, gender, body-massindex (BMI), co-morbidities, CPAP use and reasons for failure, Epworth Sleepiness Score (ESS), sleep study data, spirometry data and average maximum nightly compliance were recorded.

Results We included 52 patients with OSA who required CPAP $>15 \mathrm{cmH} 20$ (71\% male, age 58 (15) years, BMI 42.6 (10.1) kg/m2, AHI 51.1 (30.4)/hour); 62\% had respiratory co-morbidities affecting nocturnal breathing (COPD, OHS). CPAP was used for 199 (106-477) days prior to referral. Reasons for CPAP failure were intolerance of pressures (23\%), uncontrolled symptoms (23\%), mask problems (21\%), adverse effects (13\%), claustrophobia (8\%), co-morbidities $(8 \%)$, and other issues (4\%). Lower expiratory positive airway pressures (EPAP) were needed to control nocturnal breathing compared to CPAP (10 (8-12) vs 16.8 (15.7-19.2) $\mathrm{cmH} 20, \mathrm{p}=0.001)$ and patients achieved better adherence (7.0 (4.0-8.5) vs $2.5(1.6-6.7)$ hours/night, $\mathrm{p}=0.028)$ and better symptom control (ESS 4.0 (1.0-7.0) vs 10.0 (6.0$17.0)$ points, $\mathrm{p}=0.039$ ) on BPAP.

Discussion In patients with OSA with limited success on CPAP therapy, BPAP is better tolerated and achieves sufficient respiratory and symptom control.

\section{P022 \\ AN INVESTIGATION INTO THE EFFECTS OF A 20- MINUTE NAP-OPPORTUNITY ON COGNITIVE FUNCTION IN STUDENTS AGED 18-30}

${ }^{1}$ Suraiya Haddad, ${ }^{1}$ Rezwana Rahman, ${ }^{1}$ Felyx Wong ${ }^{*},{ }^{1}$ W Jackson, ${ }^{1,2}$ Mary Morrell. ${ }^{1}$ Imperial College London, London, UKi ${ }^{2}$ Academic Unit of Sleep and Ventilation, National Heart and Lung Institute, Imperial College London, Royal Brompton and Harefield National Health Service Foundation Trust

\subsection{6/bmjresp-2019-bssconf.22}

Introduction Sleep-deprivation is increasing in prevalence and compensatory napping is becoming common practice. Little is known about the cognitive benefits derived from a nap in sleep-deprived individuals, or within the student population. Furthermore, few studies have investigated the cognitive benefits of napping using the incongruent Stroop test. This study aimed to investigate the effects of a nap-opportunity on cognitive function in individuals aged 18-30.

Methods A randomised, crossover study was conducted. Participants were recruited from Imperial College London and were allocated to either control (no nap-opportunity) or intervention (20-minute nap-opportunity) groups.

The incongruent Stroop test was used to measure reaction time (RT) and percentage accuracy, which were used as markers of cognitive function. $\Delta \mathrm{RT}$ and $\Delta$ percentage accuracy, between control and nap-opportunity groups were compared. Paired and unpaired t-tests were used for the analysis of RT. Wilcoxon signed-rank and Mann-Whitney-U tests were used to test the percentage accuracy. The study was approved by the Imperial College Medical Education Ethics Committee.

Results Participants $(n=29)$ had a mean age of $20(S D \pm 1.4)$ years, $55 \%$ male. In those who were acutely sleep-deprived, the RT improved in the intervention group following a 20 minute nap-opportunity, compared to the control group $(\mathrm{p}=0.01$; figure 1$)$. Overall, no significant difference in $\Delta$

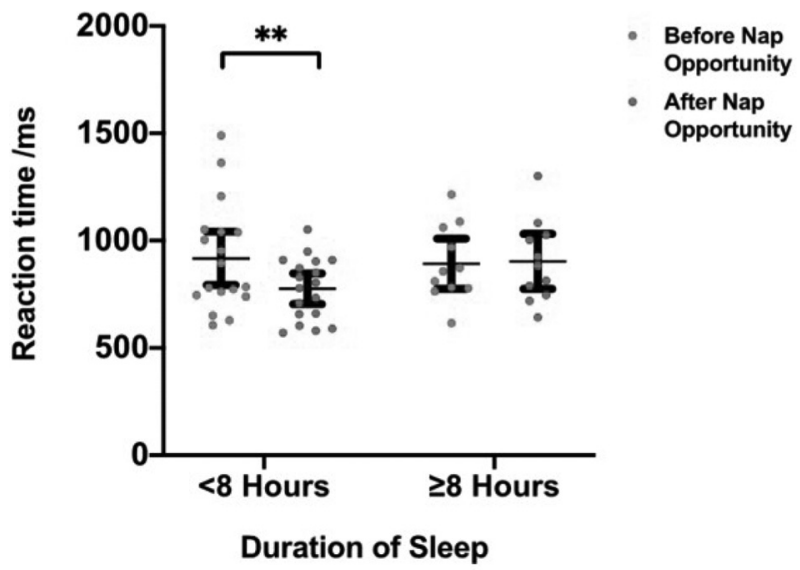

Abstract P022 Figure 1 Dot plot showing reaction times in incongruent Stroop test, measured before and after a 20 minute napopportunity in individuals who slept for $<8 \mathrm{hrs}$ the night before the test $(n=18)$ and $=8 \mathrm{hrs}$ the night before the test $(n=11)$. Data presented as mean $\pm 95 \% \mathrm{Cl}$. Paired t-test was used to compare reaction times before and after nap-opportunity. Unpaired t-tests were used to compare baselines and endpoints of the two groups. Significant change in reaction time was observed following nap-opportunity in individuals who slept $<8$ Hours before the test day $(p=0.01275){ }^{*}=P<0.05$,

${ }^{* *}=\mathrm{P}<0.01,{ }^{* * *}=\mathrm{P}<0.001$ 
percentage accuracy was observed between the control and nap-opportunity groups $(\mathrm{p}=0.31)$.

Conclusions The main finding of this study was that the sleep-deprived group derive greater benefit in cognitive function from a 20-minute nap-opportunity. Future studies could investigate the relationship between the amount of sleep-deprivation and the magnitude of benefit derived from a nap-opportunity. Moreover, the impact of napping in individuals who are chronically sleep-deprived should also be explored.

\section{P023 A SYSTEMATIC REVIEW AND META-ANALYSIS OF PHARMACOLOGICAL INTERVENTIONS FOR SLEEP PROBLEMS IN CHILDREN AND ADOLESCENTS WITH AUTISM SPECTRUM DISORDER}

${ }^{1}$ Raghad Alonazi, ${ }^{1}$ Rahaf Alonazi, ${ }^{1}$ Amna Al-Ahmed, ${ }^{1}$ Hajar Arnous, ${ }^{2}$ Faisal Alotaibi, ${ }^{3}$ Hetaf Alammar*. ${ }^{1}$ Medical intern at Dar Al Uloom University, Riyadh; ${ }^{2}$ Paediatrician at King Fahad Medical City, Riyadh; ${ }^{3}$ Assistant professor in psychology at Shaqra University, Shaqra

\subsection{6/bmiresp-2019-bssconf.23}

Aim The aim of our systematic review and meta-analysis was to synthesise the high-quality evidence on the efficacy and safety of pharmacological interventions to manage sleep problems in children and adolescents with autism spectrum disorder (ASD).

Methods The studies we included here were (RCTs) looking at the efficacy and safety of pharmacological intervention for sleep problems in children and adolescents with ASD. The studies had to include objective sleep measures and/or subjective sleep measures. They all dealt with pharmacological interventions, with medications including melatonin and antipsychotics, and they all compared these drugs with placebos. We used four databases: ERIC, ProQuest, PubMed and Ovid Medline. We assessed the papers in accordance with the Cochrane risk of bias tool.

Results We found six studies that used RCT designs. Five of them used melatonin, and one study used an antipsychotic. In studies using melatonin, a meta-analysis revealed that there was a large effect size of using melatonin in reducing sleep latency in children and adolescents with ASD. The overall effect size was -0.87 (95\% confidence interval [CI]) -1.11, $0.63, Z=7.11 \quad(P<0.00001)$. In terms of antipsychotics, our review revealed that the antipsychotic resperidon was safe and effective in managing sleep problems in children and adolescents with ASD. Both melatonin and resperidon improved sleep latency and reduced night-time awakenings. However, mild side effects were reported in a small number of participants (figure 1).
Discussion Although the quality of the evidence in these studies was moderate according to the GRADE approach, both melatonin and resperidon seem to be safe and effective in improving sleep in children and adolescents with ASD. Some side effects have been observed in a small number of children. Observing and recording side effects is crucial to control medication doses.

\section{P024 CHARACTERISING SCHOOL-AGE CHILDREN'S SLEEP IN SHAQRA PROVINCE, SAUDI ARABIA}

${ }^{1}$ Hetaf Alammar*, ${ }^{2}$ Jane Blackwell. ${ }^{1}$ Assistant Professor in Psychology at Shaqra University, Shaqra; ${ }^{2}$ Postdoctoral Researcher, Child Oriented Mental health Intervention Centre (COMIC), Leeds and York Partnership NHS Foundation Trust and The University of York, York

\subsection{6/bmiresp-2019-bssconf.24}

Introduction As no previous studies have characterised the sleep of school-age children in Shaqra Province, Saudia Arabia, the aim of the current research was to assess the frequency of behaviours associated with common paediatric sleep difficulties in this population using the Arabic version of the Children's Sleep Habits Questionnaire.

Methods The Children's Sleep Habits Questionnaire was used to measure self-reported and parent-reported bedtime resistance, sleep onset delay, sleep duration, sleep anxiety, night wakings, parasomnias, sleep disordered breathing and daytime sleepiness. ${ }^{3}$ Families were recruited through six schools and parents were asked to complete the questionnaire on behalf of children aged 7-12 years old, whereas adolescents completed the self-reported version.

Results 150 females and 139 males aged between 7-17 years old were recruited (see table 1). 92\% of the children and adolescents had a score of 41 or above indicating that they have a clinically significant sleep problem $(89 \%$ of males and 95\% of females).

Results also indicated that there was a significant difference between males and females in secondary school in total score of CSHQ and sub score (sleep duration, sleep anxiety and sleep parasomnia). In addition, there was a significant difference between males and females in intermediate school in sub score of CSHQ in bedtime resistance, sleep anxiety and sleep disorder breathing (see table 2).

Discussion The current study highlights the high prevalence of clinically significant sleep problems in this population. The results are consistent with previous research which suggests that children from Saudi Arabia sleep less than children in other countries, ${ }^{1}$ that males have longer sleep durations compared to females and that females report more daytime sleepiness compared to males. ${ }^{2}$ The results suggest that increasing

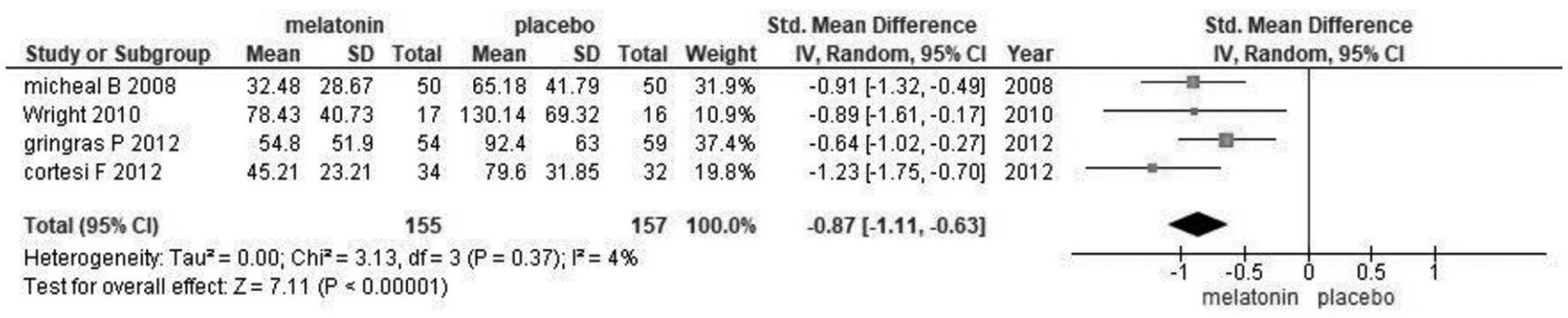

Abstract P023 Figure 1 Meta-analysis of the efficacy of using melatonin in improving sleep latency in children and adolescents with ASD 